\title{
A novel 4-sensor fast-response aerodynamic probe for non-isotropic turbulence measurement in turbomachinery flows
}

\author{
Original article \\ Article history: \\ Accepted: 15 March 2018 \\ Published: 17 May 2018

\section{D) Check for updates \\ *Correspondence:} \\ ACC: achasogl@ethz.ch
}

Peer review:

Single blind

\section{Copyright:}

ㄷ 2018 Chasoglou et al. @ This is an open access article distributed under the Creative Commons Attribution License (CC-BY 4.0), which permits unrestricted use, distribution and reproduction in any medium, provided the original work is properly cited and its authors credited.

\section{Keywords:}

non-isentropic turbulence measurements; multi-sensor fast response probes; unsteady flow

\section{Citation:}

Chasoglou A. C., Mansour M., Kalfas A. I., and Abhari R. S. (2018). A novel 4-sensor fast-response aerodynamic probe for nonisotropic turbulence measurement in turbomachinery flows. Journal of the Global Power and Propulsion Society. 2: 362-375.

https://doi.org/10.22261/JGPPS.UALS07

\author{
Alexandros C. Chasoglou ${ }^{1, *}$, Michel Mansour ${ }^{2}$, Anestis I. Kalfas ${ }^{3}$, \\ Reza S. Abhari ${ }^{1}$ \\ ${ }^{1}$ Laboratory for Energy Conversion, ETH Zurich, Department of \\ Mechanical and Process Engineering, Sonneggstrasse 3, Zurich, \\ $\mathrm{CH}-8092$, Switzerland \\ ${ }^{2}$ Limmat Scientific AG, 6300 Zug, Switzerland \\ ${ }^{3}$ Department of Mechanical Engineering, Aristotle University of \\ Thessaloniki, 54124, Thessaloniki, Greece
}

\begin{abstract}
In modern computational studies for turbomachinery applications, time, length scales and isotropy of turbulent structures are important for representative modelling. To this end, experimental data are essential to validate the numerical tools. The current article presents the development and application of a newly designed 4-sensor Fast Response Aerodynamic Probe (FRAP-4S) enabling time-resolved measurement of the threedimensional unsteady flow velocity vector in turbomachines. The miniature multi-sensor probe demonstrates a $4 \mathrm{~mm}$ probetip. In the first part of this article the design, manufacturing and calibration results of the FRAP-4S are presented in detail. To assess the newly developed probe accuracy, comparison against traditional instrumentation developed at the Laboratory for Energy Conversion is also provided. In the second part of this work, measurements are performed at the rotor exit of a oneand-a-half stage, unshrouded and highly-loaded axial turbine configuration. The results showed increased level of unsteadiness and turbulence levels with peak-to-peak fluctuation from 5 to $35 \%$. More importantly, in some regions stream-wise unsteadiness was found to be ten times higher, compared to the cross-wise components, an indication of the high degree of anisotropy.
\end{abstract}

\section{Introduction}

Considering the unsteady flow in an axial turbomachine, the frequency spectrum of the fluctuations is in the range of a few hertz up to several multiples of the blade passing frequency. For gaseous-flows in turbomachines, frequencies up to $50 \mathrm{kHz}$ are, in most cases, of practical interest (Kupferschmied et al., 2000a). The time and length scales of the flow structures vary up to six orders of magnitude, due to the diverse nature of the originating mechanism (Abhari, 2006). Periodic fluctuations 
arise due to regular wake passing or other secondary and leakage flow features, or even shocks (Denton, 1993). On the other hand, stochastic fluctuations can be attributed to turbulence, to unsteady transition, separation of boundary layers, etc. In order to understand the loss mechanisms under these conditions, experimental work and computational tools should come together to evaluate the complex flow structures. A comprehensive review of measurement techniques for unsteady turbomachinery flows can be found in Ainsworth et al. (2000); Sieverding et al. (2000).

Despite the advancements in numerical modelling and computational tools, turbulence models are mainly based on a series of assumptions or simplifications, which may be mathematically plausible but do not necessarily accord with the actual physics in a turbulent flow. Nevertheless, turbulence modelling is inherently connected to the design and development process in internal turbomachinery applications. In the past, numerical tools were validated through experimental investigations in simplified test rig geometries, while lately the interest has shifted towards experimental data acquisition under realistic complex flow conditions (Camp and Shin, 1995; Porreca et al., 2007; Lengani et al., 2012).

In the majority of experimental studies, the turbulence quantities are derived from hot-wire anemometry measurements. However, hot-wire probes suffer from their inherent fragility and loss of sensitivity at high Mach numbers, which makes them suitable for lightly loaded, low-speed turbomachines. To overcome this issue, fast-response pressure probes equipped with embedded piezo-resistive pressure sensors have been developed for highly loaded and high-speed turbomachines. Heneka was probably the first to attempt deriving turbulence quantities with a 4-hole wedge unsteady probe (Heneka, 1983). This probe was used for turbulence measurements in both freejet and high speed compressor by Ruck et al. (Ruck, 1988; Ruck and Stetter, 1990). Gossweiler (1993) developed a miniature 4-sensor Fast Response Aerodynamic Probe (FRAP-4S), which was used to derive turbulence parameters in a pipe flow. In another work, Köppel (2000) measured time-resolved flow quantities using a 1-sensor FRAP, assuming isotropic conditions and incompressible flow. Porreca et al. (2007) presented a methodology to infer turbulence quantities, for incompressible flows, in all three spatial directions, using the standard FRAP-2S under virtual 4-sensor mode (V4S) (Pfau et al., 2002). Similarly, yawing a 1-sensor unsteady probe in different positions in order to derive the turbulent parameters was also demonstrated in Persico et al. (2008).

In the context of this work, the term 'multi-sensor' will refer to unsteady probes with more than two sensors. In early designs, unsteady probes had surface mounted sensors (Kerrebrock, 1972; Bubeck and Wachter, 1987). To avoid sensor damage due to particle impact, the latter probes' sensors were also coated. Furthermore in Bubeck and Wachter (1987), the probe design demonstrated a pneumatic hole to measure the total pressure in a time-average manner, a design option based on space limitations. The predecessor of Budecks' probe was the wedge type probe designed by Heneka (1983). The probe developed by Gossweiler (1993) represents the most compact unsteady probe of its kind. To achieve a probe tip diameter of $2.5 \mathrm{~mm}$, the yaw sensitive sensors had to be shifted in respect to the centre hole, thus resulting in a slightly larger sensing area than desired. Nevertheless, this design was abandoned and never used for turbomachinery measurements. It is worth noting, that the majority of the probes incorporate absolute pressure transducers, which limits the operating range of the proposed designs. In Brouckaert (2007), an unsteady yaw probe was presented. This design incorporates interesting features, like the rectangular sensing ports to enhance the frequency response of the sensors and an airbleed design. This latter feature was used to supply air at the trailing edge of the probe, to stabilize the probe-induced wake and reduce errors due to unsteady lift and dynamic stall, which are present in the current probe due to its asymmetric cross-section profile.

Finally, in Zimmermann and Wirsum (2016) an L-shaped 5-sensor unsteady probe was presented, which is employing the commercially available Kulite multi-sensor tip. The $6.35 \mathrm{~mm}$ tip was inserted to a $8 \mathrm{~mm}$ stem part. As reported from the manufacturer, the frequency response is rather high, of the order of $100 \mathrm{kHz}$, but the resulting probe diameter and the relative large distance between the circumferential pressure taps impacts substantially the spatial resolution. Furthermore, it should be mentioned, that the rather large L-shaped tip design makes measurement in actual test rigs more challenging, since access holes for probe measurements usually range from 6 to $12 \mathrm{~mm}$.

The current article describes the development and application of a newly designed FRAP-4S enabling the simultaneously measurement of the unsteady three-dimensional flow velocity vector in 
turbomachines. In the following sections, the design, manufacturing approach and calibration of the FRAP-4S are presented in detail. Subsequently, the probe performance is compared against the traditional FRAP 2-sensor (FRAP-2S) and 5-Hole Probe, (5HP) for the assessment of its relative accuracy. Finally, a detailed analysis of the turbulence intensity distribution at the rotor exit of an axial turbine is provided and discussed.

\section{FRAP-4S probe description}

The design, manufacture and operation of the newly developed fast response aerodynamic probe is based on longstanding experience gained over the last two decades at the Laboratory for Energy Conversion (LEC) at ETH Zürich (Kupferschmied et al., 2000b; Mansour et al., 2008; Lenherr et al., 2010; Bosdas et al., 2016, 2017). In particular, the FRAP-4S is based on the well-established standard FRAP-2S. The FRAP-4S uses similar packaging and bonding solutions, but it has four miniature piezo-restive sensors embedded into the probe tip, instead of two for the FRAP-2S. The sensors were positioned underneath the sensing taps, where the sensor's membrane is protected from particle impact with a metal shield. The overall dimensions of FRAP- $4 S$ are provided in Figure 1a, along with the pressure tap notation and the sign convention in the probe frame of reference. The probe has perpendicular facing holes with a diameter of $0.35 \mathrm{~mm}$, while the included angle between the taps in the yaw plane is equal to $42^{\circ}$. The pitch sensitive sensor is positioned in the spherical tip where the tap is placed at $45^{\circ}$ in respect to the centre hole (Figure 1a).

Compared to FRAP probes developed at LEC in the past, the probe demonstrates a spherical tip head, rather than the traditional filleted cylinder. The actual probe is shown in Figure 1b. The probe tip diameter is $4 \mathrm{~mm}$, which is connected to a $6.35 \mathrm{~mm}$ diameter stem located ten tip diameters from the tip (Figure $1 \mathrm{~b}$ ). The overall length of the probe is $1 \mathrm{~m}$. The main design challenges of the newly developed FRAP-4S were to accommodate the installation of four piezo-resistive pressure sensors within a miniature tip, while maintaining the required measurement bandwidth, sensitivity and robustness for applications in turbomachinery flows.

\section{Piezo-resistive sensor static calibration}

The FRAP-4S employs four miniature silicone piezo-resistive sensors, which incorporate the Wheatstone bridge configuration (Figure 1c). Similar to the previously developed FRAP probes, the pressure-sensitive chips are operated under constant excitation current $I_{e}$. The induced excitation voltage $U_{e}$ is primarily temperature dependent, while the resultant voltage output $U$ is strongly dependent on the differential pressure induced athwart the sensor membrane.

For this study, the piezo-resistive sensors are calibrated over a differential pressure range and static temperature range of $50-1,000 \mathrm{mbar}$ and $20-120^{\circ} \mathrm{C}$ respectively. The resulting $U_{e}$ and $U$ signal variation are shown indicatively in Figure 2 for one of the embedded sensors. Despite the increase in sensors' packaging density, the quality of the static calibration results remains similar to the FRAP-2S (Mansour et al., 2008). Moreover, the probe was subjected to a 40-hour thermal cycling test, in the

a

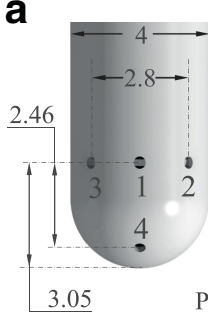

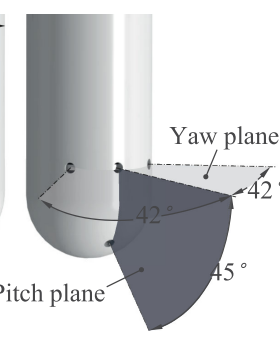

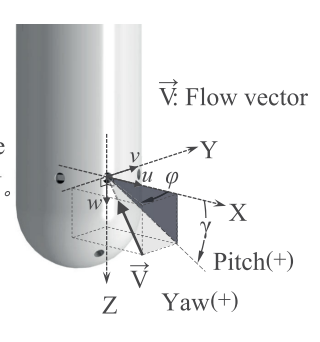

b

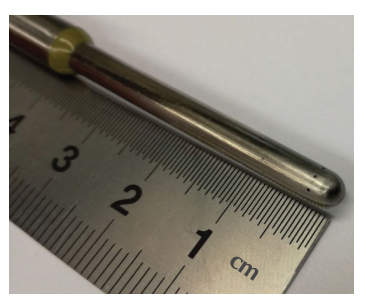

C

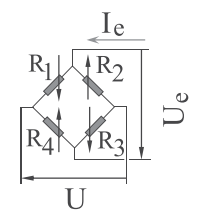

Figure 1. (a) FRAP-4S probe tip schematic, relevant dimensions (in $\mathrm{mm}$ ) and port annotation; (b) Manufactured probe tip; (c) Schematic of the Wheatstone bridge configuration. 

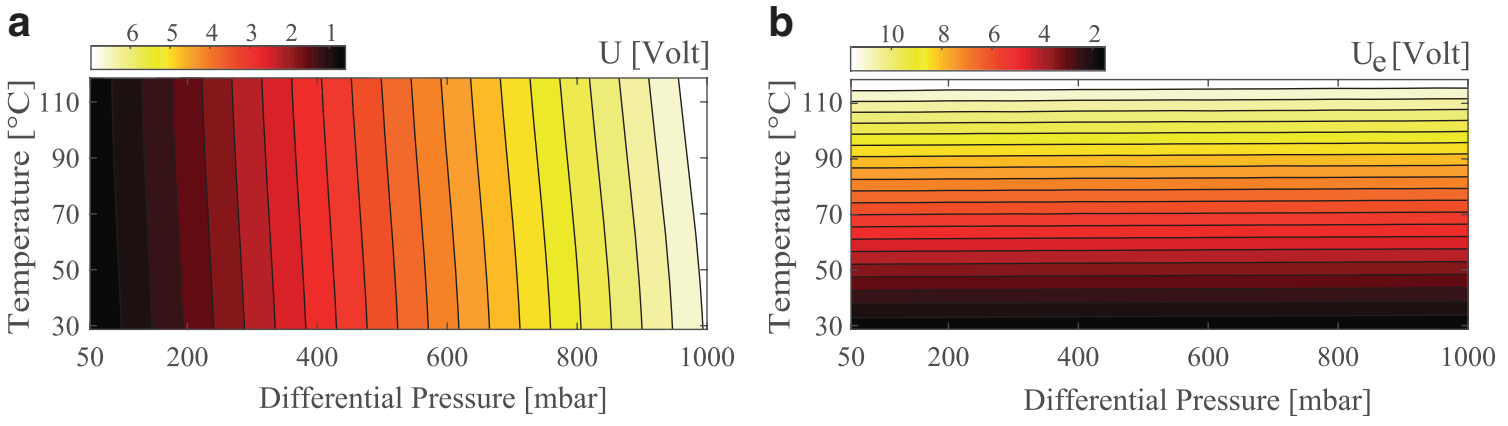

Figure 2. Yaw sensor (sensor 1) calibration curves for pressure - $U$ (a) and temperature - Ue signal (b).

aforementioned operating conditions. This long-duration test showed no significant thermal drift for the sensors, while also maintaining excellent repeatability.

\section{Steady aerodynamic probe calibration}

The FRAP-4S probe is calibrated in the free-jet facility of the LEC at ETH Zürich, more details regarding the operation of the facility can be found in Kupferschmied et al. (2000b). The calibration procedure is based on traditional methods described in Kupferschmied et al. (2000a) and Johansen et al. (2001). A set of non-dimensional calibration coefficients is formulated from the probe's pressure signal, total and static pressure of the flow. The coefficients are summarized in Equation 1 where $K_{\varphi}$ and $K_{\gamma}$ are the flow angle coefficients, $K_{t}$ and $K_{s}$ the total and static pressure coefficients respectively and $\mathrm{p}_{\mathrm{m}}=\left(\mathrm{p}_{2}+\mathrm{p}_{3}\right) / 2$.

$$
\begin{aligned}
& \mathrm{K}_{\varphi}=\frac{\mathrm{p}_{2}-\mathrm{p}_{3}}{\mathrm{p}_{1}-\mathrm{p}_{\mathrm{m}}} \quad \mathrm{K}_{\gamma}=\frac{\mathrm{p}_{1}-\mathrm{p}_{4}}{\mathrm{p}_{1}-\mathrm{p}_{\mathrm{m}}} \quad \text { (a) } \\
& \mathrm{K}_{\mathrm{t}}=\frac{\mathrm{p}_{\mathrm{tot}}-\mathrm{p}_{1}}{\mathrm{p}_{1}-\mathrm{p}_{\mathrm{m}}} \quad \mathrm{K}_{\mathrm{s}}=\frac{\mathrm{p}_{1}-\mathrm{p}_{\mathrm{stat}}}{\mathrm{p}_{1}-\mathrm{p}_{\mathrm{m}}} \quad \text { (b) }
\end{aligned}
$$

In the present work, the probe was calibrated for Mach numbers of 0.3 and 0.5 at $30^{\circ} \mathrm{C},\left(\operatorname{Re}_{D} 25 \cdot 10^{3}\right.$ and $43 \cdot 10^{3}$ respectively) conditions. The calibration maps are provided in Figure 3. In order to assess the probe's aerodynamic performance, FRAP- $4 \mathrm{~S}$ is calibrated to $\mathrm{a} \pm 32^{\circ}$ in yaw and pitch. The results have shown that the sensitivity of the probe pitch flow angle coefficient drops drastically for positive pitch angles, $\gamma \geq 20^{\circ}$ as flow tends to separate on pressure port 4 for pitch incidence above $22^{\circ}$. The quality and the symmetricity of the obtained calibration curves proves the good accuracy during the manufacturing process.

\section{Probe dynamic response}

The frequency response of the pressure signal depends on the eigen-frequency of the pneumatic cavity formed between the upper surface of the sensing hole and the sensor's membrane. The eigen-frequency
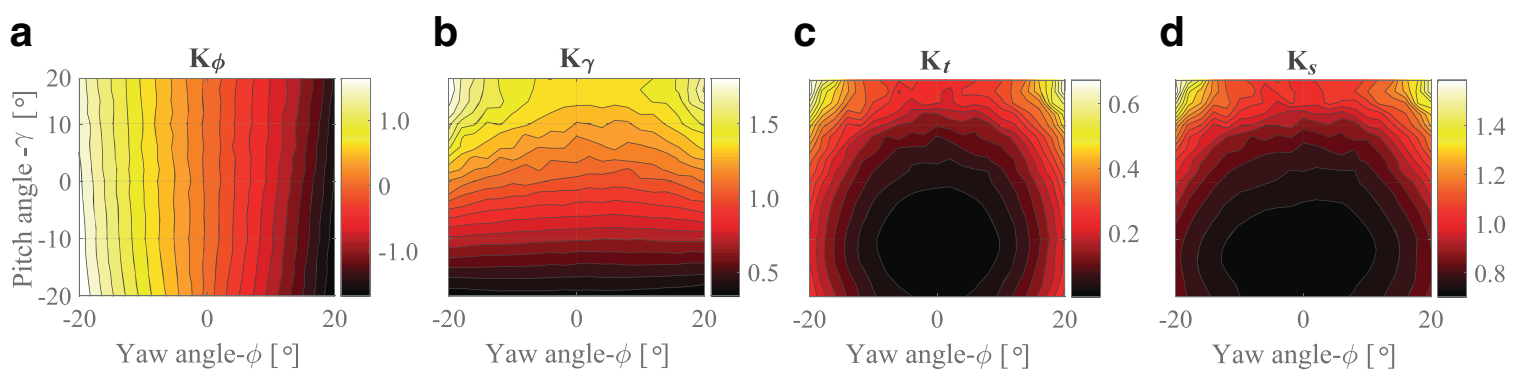

Figure 3. FRAP-4S Aerodynamic coefficients for (a) yaw, (b) pitch angles, (c) total and (d) static pressure at $M=0.3$. 
of the cavity was obtained in the freeject facility based on the methodology described by Pfau et al. (2002). Total pressure fluctuations induced by a fine turbulence mesh grid at the exit of the freejet are sufficient to acoustically excite the pneumatic cavity over a broad range of frequencies. The results of this test show that all the sensors positioned in the yaw plane demonstrate similar behaviour with eigen-frequencies ranging from 80 to $85 \mathrm{kHz}$. These small discrepancies arise from manufacturing tolerances and small differences that occur during sensor packaging. This observation indicates the repeatability of the manufacturing process. The eigen-frequency of the pitch sensor $\left(P_{4}\right)$ was lower, in the range of $65 \mathrm{kHz}$ due to an increased tube length of this pressure tap compared to the rest sensors. Mechanical integrity-related issues imposed this design option. Nevertheless, it was found that for all sensors the amplitude is flat up to at least $45 \mathrm{kHz}$, which determines the cut-off frequency and the measurement bandwidth of the FRAP- 4 S probe. The obtained measurement bandwidth remains similar to the traditional FRAP-2S (Mansour et al., 2008), which was one of the main design requirements of the FRAP-4S. It should be added that no digital compensation in terms of measurement bandwidth was performed for the probe used in this study. Deriving the transfer function of the probe through shock-tube testing will allow doubling of the available measurement bandwidth and was planned at a later stage. Furthermore, the flow temperature can be measured in a time-averaged manner, with bandwidth in the range of $10 \mathrm{~Hz}$ due to thermal inertia of the embedded sensor.

\section{Dynamic effects}

Fast response aerodynamic probes used for measurements in turbomachines are subjected to dynamic flow conditions. Strong velocity gradients and flow angle fluctuations are predominant in these type of flows and have shown to affect the accuracy of the measurement probe (Humm et al. (1994)). Furthermore, in turbomachinery probe flow measurements the majority of the errors are proportional to the probe size and probe geometry (Kupferschmied et al., 2000a). A non-dimensional parameter related to the degree of unsteadiness in the flow, is the reduced frequency $k$.

$$
k=\frac{f \cdot \mathrm{D}}{\mathrm{C}}
$$

In Equation 2, $f$ represents the excitation frequency, i.e., the Blade Passing Frequency (BPF), $D$ the probe tip diameter and $C$ is the local free stream velocity. According to Humm et al. (1995), the errors induced by dynamic flow phenomena are significantly reduced for cylindrical stem probes and for reduced frequencies below a critical value of $k_{\text {crit }}=0.1$. Thus, the newly developed FRAP-4S has a diameter of $4 \mathrm{~mm}$ to reduce the impact on the unsteady measurement accuracy. Figure 4 illustrates the operating envelope of the probe, in terms of $B P F$ and flow velocity, while also indicating the flow conditions in this study. The dashed lines indicate the critical value of $k_{\text {crit }}=0.1$ and the $k=0.2$ where lock-on of the Kármán vortex shedding occurs for the Reynolds number range of $25 \cdot 10^{3}<R e_{D}<43 \cdot 10^{3}$, based on probe diameter.

In order to obtain additional information regarding the dynamic effects on this new probe design, the pressure signal fluctuations were evaluated at different locations in the measurement plane at the exit of the rotor. In Figure 5 the frequency spectrum of the raw voltage signal $U$ is provided for both FRAP-

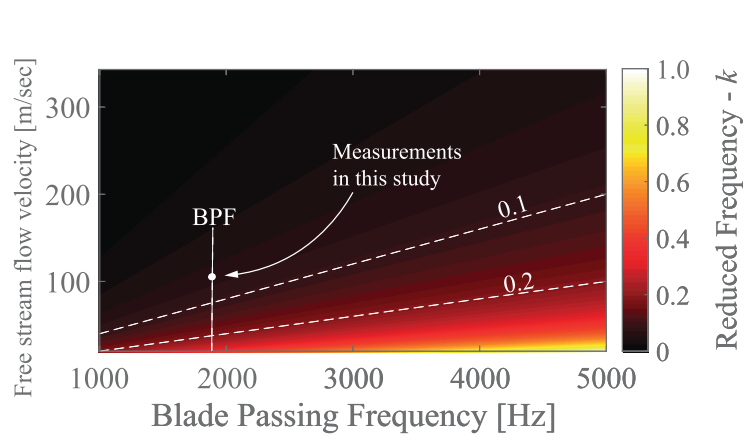

Figure 4. Reduced frequency versus BPF and free stream flow velocity.
$4 \mathrm{~S}$ and FRAP-2S. These pressure signals refer to the center sensor, port 1 , with both probes positioned at mid-span and in the middle of the stator pitch. There is a good overall agreement, while no frequency content related to amplitude amplification due to vortex shedding can be readily found, except of what is related to rotational speed and blade passing frequency modulation (Figure 5b). Slight peaks around the fundamental blade passing frequency are separated by a $\Delta f=45 \mathrm{~Hz}$. Similar behaviour was observed by Regina (2015) is attributed to the interaction of the blade passing with the possible 
a

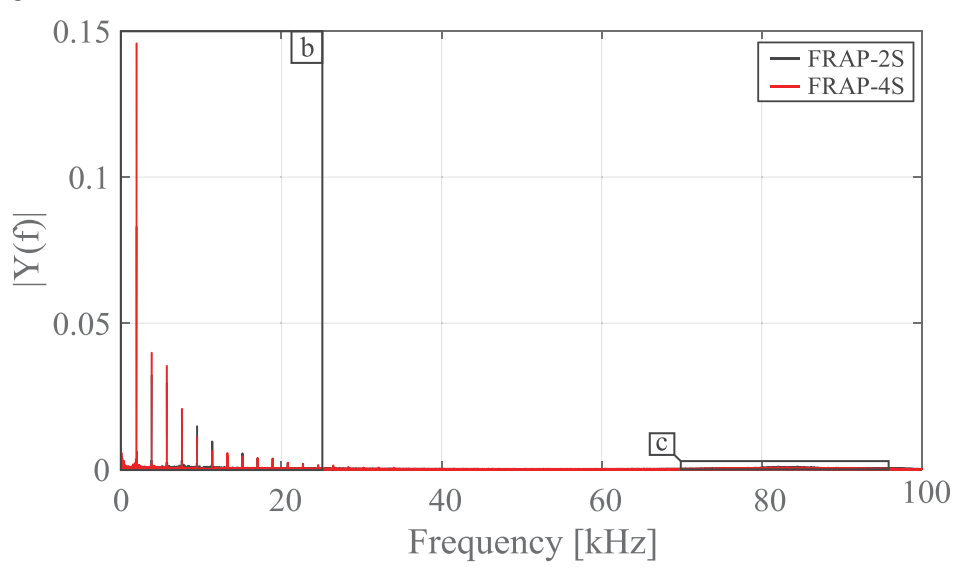

b

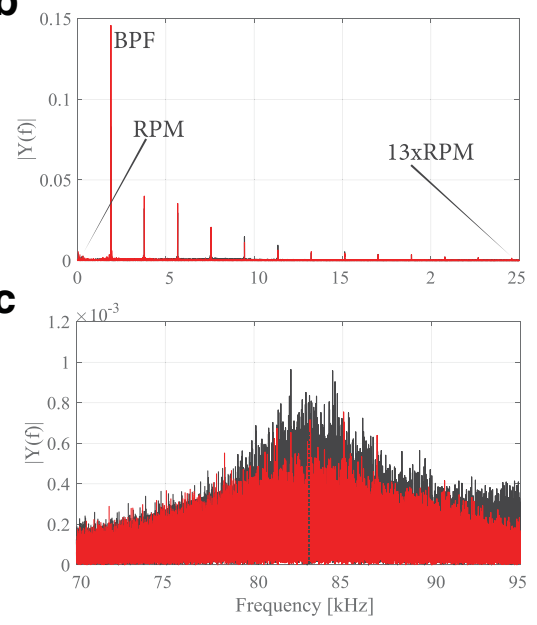

Figure 5. Frequency spectrum of raw pressure signals for FRAP-2S' and FRAP-4S' sensor 1 in the middle of the blade passage.

out-of-roundness effect. A peak around $84 \mathrm{kHz}$ for both probes was found, which is related to the eigen-frequency of the pneumatic cavity formed between sensing hole and sensor membrane (Figure 5c). For completeness, it should be highlighted, that FRAP-2S has a tip diameter equal to $1.8 \mathrm{~mm}$, resulting in reduced frequencies below 0.035 and 0.018 respectively for the same operating conditions. Nevertheless, the turbulent power spectra comparison with the FRAP-2S confirms the capability of the FRAP-4S to measure stochastic pressure fluctuations with the same degree of accuracy as the FRAP-2S up to $45 \mathrm{kHz}$.

\section{Experimental set-up and methodology}

\section{Research facility}

A set of measurements to demonstrate the capabilities of the 4-sensor fast response aerodynamic probe was performed in the axial research turbine test facility "LISA". Figure 6a gives an overview of the facility. For this study, the rig was configured with an one-and-a-half stage, high-pressure turbine representative arrangement. Moreover, the three blade rows were designed with non-axisymmetric end-wall contouring at the hub, and end-wall contouring at the tip for the first stator. The rotor incorporates an unshrouded design and tip clearance is $1 \%$ of the blade span. The measurement plane is indicated in the schematic in Figure 6b.

The highly loaded turbine operates at moderate speeds and low temperatures. The rotational speed of the turbine is kept constant by a DC generator with an accuracy of $\pm 0.02 \%( \pm 0.5 \mathrm{rpm})$. Furthermore, the turbine inlet total temperature is maintained constant with an accuracy of $\pm 0.3 \mathrm{~K}$, with the use of a heat exchanger at the inlet. A detailed description of the turbine design can be found in Behr et al. (2007). The main operational parameters of the facility are summarized in Table 1. For this work, measurements were performed at the exit of the rotor, over one stator pitch. A total of 41 traverses was performed in the circumferential direction (every $2.5 \%$ of the pitch), while 37 radial positions were taken per traverse. The radial points were clustered towards hub and tip and cover a range from 7 to $99.5 \%$ span. The measurement grid of total 1,517 points required approximately four hours for FRAP-4S. The same measurements for FRAP-2S need close to 9.5 hours, due to V4S operation. The FRAP data were acquired with $200 \mathrm{kHz}$ sampling rate and 24 Bit resolution. This resulted in a total of 105 samples per rotor blade passage. An ensemble of 85 revolutions are then kept for phase-lock averaging and to perform statistically reliable analysis. 

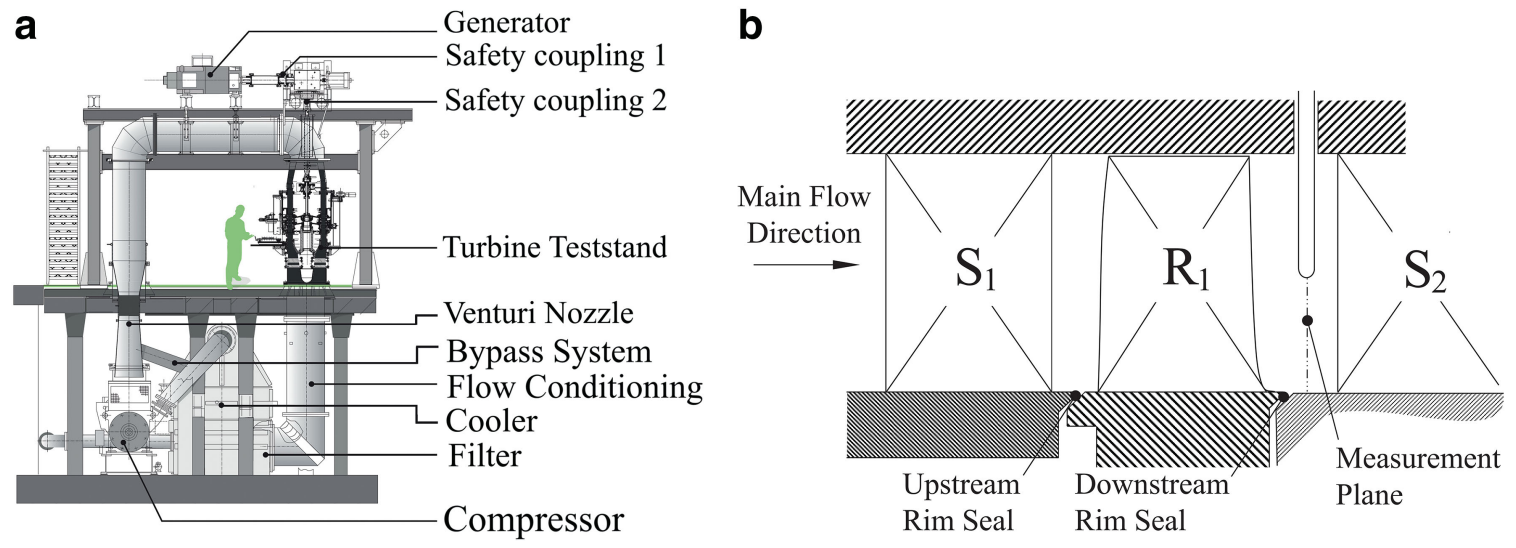

Figure 6. Schematic of "LISA" axial turbine facility (a); Cross-section of the test segment (b).

Table 1. Geometrical features and operating conditions of 1.5 stage axial turbine LISA.

\begin{tabular}{l|c|c}
\hline Rotational Speed & $2700 \pm 0.5$ & {$[\mathrm{rpm}]$} \\
\hline Pressure ratio & $1.65 \pm 0.1 \%$ & {$[-]$} \\
\hline Inlet total temperature & $326.7 \pm 0.3$ & {$[\mathrm{~K}]$} \\
\hline Capacity & $150.9 \pm 0.1$ & {$\left[\frac{\mathrm{kg} \cdot \sqrt{\mathrm{K}}}{\mathrm{s} \cdot \mathrm{bar}}\right]$} \\
\hline Aspect ratio $\left(\mathrm{S}_{1} / \mathrm{R}_{1} / \mathrm{S}_{2}\right)$ & & {$[-]$} \\
\hline Blade count $\left(\mathrm{S}_{1} / \mathrm{R}_{1} / \mathrm{S}_{2}\right)$ & $0.89 / 1.21 / 0.83$ & {$[-]$} \\
\hline Mach at exit $\left(\mathrm{S}_{1} / \mathrm{R}_{1} / \mathrm{S}_{2}\right)$ & $36 / 42 / 36$ & {$[-]$} \\
\hline Chord based Reynolds number & $0.53 / 0.27 / 0.48$ & {$\left[\times 10^{5}\right]$} \\
\hline
\end{tabular}

\section{Data reduction}

Using the Reynolds decomposition, the time dependent variables in a turbulent flow can be divided into periodic and stochastic components. With this definition the absolute flow velocity in the probe relative frame of reference can be decomposed into the Phase-Locked-Averaged (PLA) periodic component $\bar{c}$ and the non-deterministic, aperiodic component due to turbulence $c^{\prime}$.

$$
\mathrm{c}(\mathrm{t})=\overline{\mathrm{c}}+\mathrm{c}^{\prime}(\mathrm{t})
$$

The turbulence intensity level $T u$ is defined using the stochastic component of the velocity vectors as defined in Equation 4, following the notation from Figure 1a.

$$
\mathrm{Tu}=\sqrt{\frac{{\overline{\mathrm{u}^{2}}}^{2}+\overline{\mathrm{v}}^{2}+\overline{\mathrm{w}}^{\prime 2}}{\overline{\mathrm{s}} \overline{\mathrm{c}}^{2}}}
$$

The stochastic unresolved part is computed from the difference between the raw signal and the PLA component. FRAP- $4 S$ requires no additional post processing and the non-deterministic component can be readily found. 


\section{Further instrumentation}

In order to further validate the time-averaged and dynamic measurement accuracy of the newly developed FRAP-4S, comparison with the traditional 5HP (Treiber et al., 1998) and FRAP-2S (Porreca et al., 2007; Behr et al., 2008; Regina et al., 2013; Schädler et al., 2017) are provided in the following sections of the article. The $5 \mathrm{HP}$ is also used to derive the time-averaged flow field. Then, the FRAP-4S is positioned according to the average yaw flow angles, to assure unsteady measurements within the calibration range of the latter probe. It is worth noting that the sensing area of FRAP-4S is increased by a factor of 2, with respect to FRAP-2S. Inevitably, the minimum resolvable turbulent length scale for the two probes is different (larger for FRAP-4S), due to increased distance between the sensor ports, as also stated in Sieverding et al. (2000).

\section{Results and discussion}

The present work focuses on the applicability of multi-sensor fast response aerodynamic probes for turbomachinery applications. First, the time-averaged measurement performance of the FRAP-4S is compared to the traditional 5HP. Subsequently, the turbulent flow-field distribution at rotor exit is discussed in detail.

Figure 7 shows the span-wise distribution of the area- and circumferentially-averaged yaw angle and $C_{p t}$ measured with the 5HP and FRAP-4S at rotor exit. The flow-field can be divided in four distinct regions: the tip leakage vortex region from 100 to $90 \%$ span, the tip passage vortex region from 90 to $70 \%$ span, the pure blade wake region from 70 to $40 \%$ span and finally the region affected by the presence of the hub secondary flow structures ranging from 40 to $10 \%$ span. The absolute deviation between FRAP-4S and 5HP measurements are shown as error-bars at the left of each graph. It can be seen that the deviations in measured yaw angles are maximum in the regions of highly skewed flow, which can be attributed to the larger sensing area and flow blockage surface of the FRAP-4S compared to the 5HP. Interestingly, in the mid-span region, from 30 to $70 \%$ span, the $5 \mathrm{HP}$ and FRAP-4S measurements show a good agreement. In the uniform region from 30-50\% span the distributions are identical, while a slight increase is shown as soon as the gradient appears at $60 \%$ span. Similarly, the steep gradient at $75 \%$ span is causing the overshoot in the deviation from pneumatic data. Regarding

a $|\Delta \phi|\left[^{\circ}\right]$

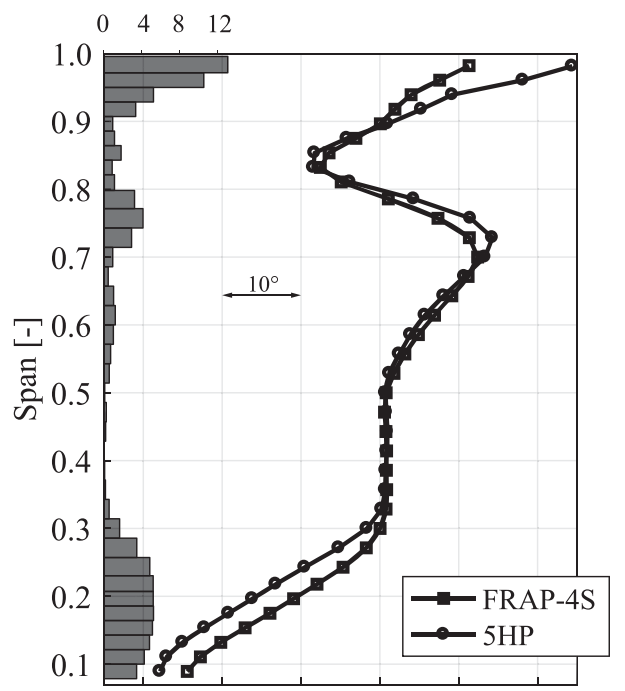

Absolute Flow Yaw Angle [ $\left.{ }^{\circ}\right]$ b $\left|\Delta C_{p t}\right|[\%]$

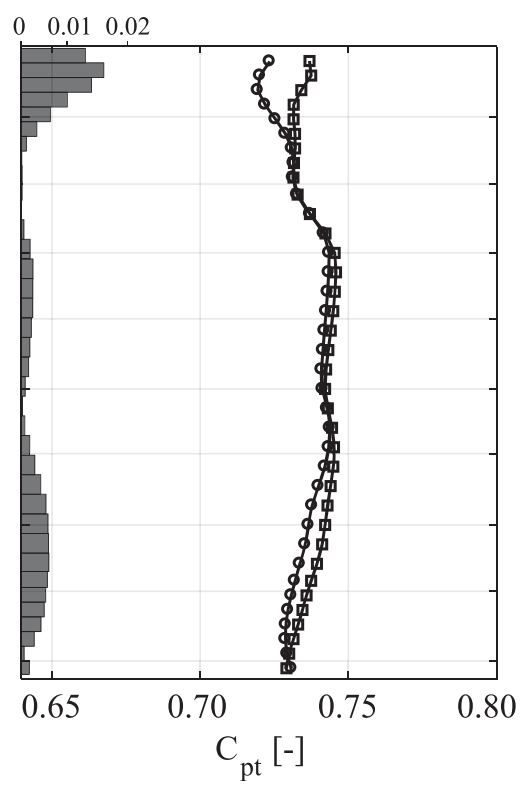

Figure 7. Area- and circumferentially-averaged (a) absolute yaw flow angle and (b) total pressure coefficient. 
the $C_{p t}$ profiles, similarly to yaw angle measurements the largest deviations occur in the regions affected by the secondary flow structures with best agreement found around mid-span. The differences on average are close to $0.5 \%$, whilst deviations of up to $2.3 \%$ can be seen at the tip, in comparison to $5 \mathrm{HP}$.

The time-averaged area plots of the measured total pressure coefficient and anisotropic turbulence are shown on Figure 8, providing an overview of the flow-field distribution across one stator pitch at the exit of the rotor. In Figure 8a, the extended total pressure deficit region (A) ranging from 75 to $100 \%$ span is directly related to the presence of the rotor tip leakage and tip passage vortices. The unshrouded rotor blades, allow the expansion of the main flow across the rotor tip gap from the pressure to the suction side, which then rolls-up into the rotor tip passage vortex. In that region, the highest levels of unsteadiness can be found with turbulence intensity levels of up to 25\% (Figure 8b). On Figure 8a, close to the hub, centred at $15 \%$ span and $35 \%$ pitch, the trace of the corner and hub passage vortices can be seen. This interaction can be identified as a local total pressure loss-core, which results in a second region (B) of increased stochastic unsteadiness, as seen on Figure 8b. The third major region (C) of total pressure deficit and elevated turbulence intensity is located between 40 and $60 \%$ span and spread over $50 \%$ of the stator pitch. This signature is most probably related to the stator wake structure, periodically chopped by the incoming rotor blade.

In this section the turbulent flow quantities and the contribution of each velocity vector to the measured level of turbulence intensity, will be discussed. A comparison is performed between isotropic and non-isotropic turbulence levels to evaluate the validity of turbulence isotropy assumption. To compute isotropic $T u_{i s o}$ only the stream-wise fluctuating component is used, based on Equation 5.

$$
\mathrm{Tu}_{\mathrm{iso}}=\sqrt{\overline{\bar{u}^{\prime}} / \overline{\mathrm{c}}^{2}}
$$

In Figure 9, the area- and circumferentially-averaged turbulence intensity profile are provided. It is clear that at this position at the exit of the rotor the assumption of isotropic turbulence does not hold. A constant discrepancy of approximatelly $2.5 \%$ across the entire span can be seen. In the regions affected by the presence of the tip passage and tip leakage vorticies at 77 and $90 \%$ span the isotropic turbulence intensity tends to overestimate levels of stocastic unsteadiness by more than $5 \%$. It should be noted that at midspan the istropic turbulence intensity shows the lowest deviation of $1 \%$ compared to the anisotropic formulation, as the contribution to the stochastic unsteadiness comes primarly form the freesteam turbulence. It can be concluded that the cross-wise velocity fluctuations are in general significantly lower compared to the stream-wise direction.

a

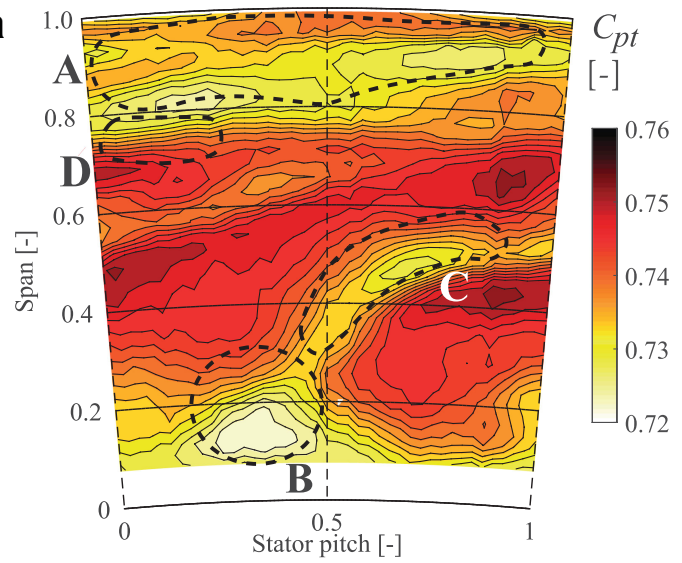

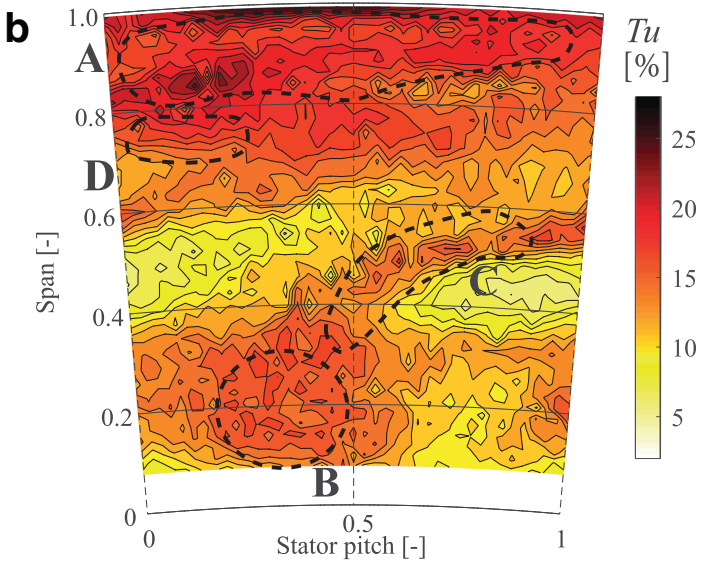

Figure 8. Time averaged (a) total pressure coefficient and (b) anisotropic turbulence intensity measured with FRAP-4S. Observer looks upstream. 


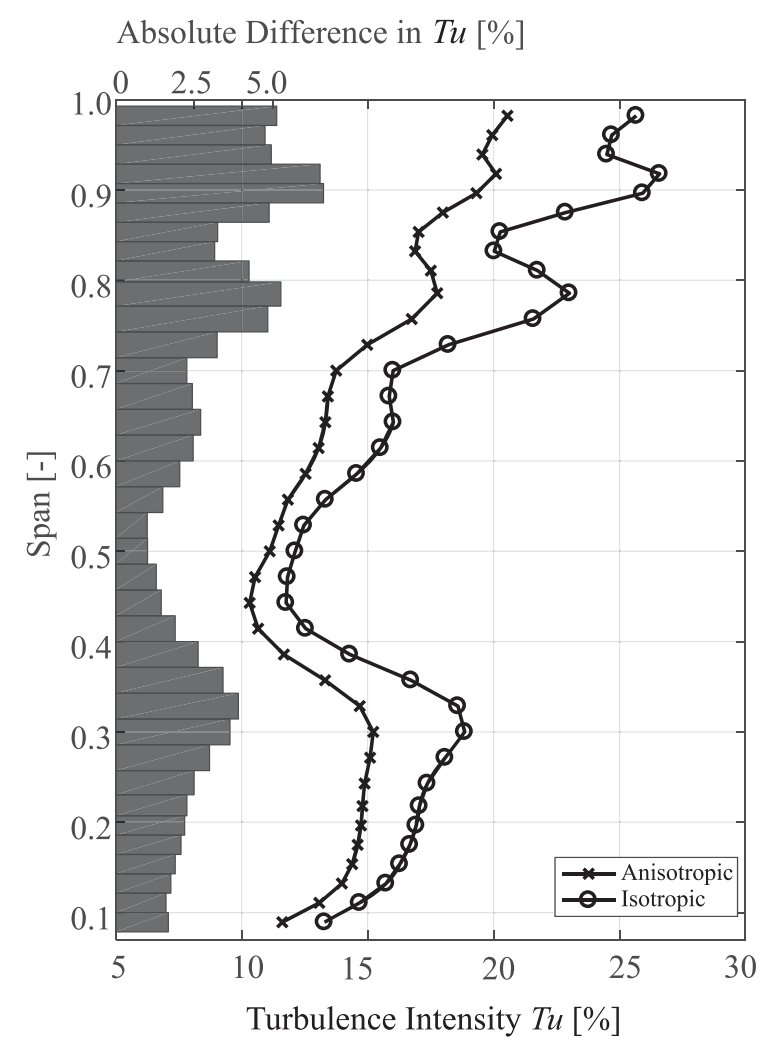

Figure 9. Area- and circumferentially-averaged turbulence intensity measured with FRAP-4S.

The time-resolved turbulence intensity across the circumference is now analysed in detail at the rotor exit at $77 \%$ span and over three rotor blade passing periods. Figure 10 presents the timespace plots for $C_{p t}$ (a) anisotropic turbulence (b), as well as the Degree of Anisotropy (DA) as defined in Equation 6.

$$
\mathrm{DA}=\frac{2{\overline{\mathrm{u}^{\prime}}}^{2}}{{\overline{\mathrm{v}^{2}}}^{2}+\overline{\mathrm{w}}^{2}}
$$

The $D A$ is used to quantify the departure from isotropy by comparing the turbulent cross-wise $(v$ and $w$ ) and stream-wise $(u)$ velocity components. As shown in Figure 10a and 10b, the presence of the rotor tip passage vortex translates into alternating regions of elevated turbulence intensity and total pressure loss. In region I, where the tip passage vortex is located, the anisotropic turbulence levels are close to $25 \%$.

The isotropic turbulence formulation leads to greater values compared to the anisotropic turbulence levels, up to more than $40 \%$ in turbulence intensity (not shown here). Furthermore, in the free-stream zone (II), turbulence is underpredicted in the isotropic case compared to the non-isotropic showing turbulence intensities ranging from 5 to $10 \%$. In the third region of interest (III), a spike-like variation of the turbulence levels can be seen and is attributed to the ingestion of the upstream stator wake by the tip passage vortex. The $D A$ plotted in Figure 10c highlights the strong peak-to-peak variations between the freestream and the secondary flow regions.

In the $D A$ distribution two regions are highlighted, the free-stream region (II) where the $D A$ decreases down to a minimum value of 0.28 and the region IV, where the stream-wise fluctuations are ten times more intense than in the cross-wise direction. In region II, the flow is only weakly affected by secondary flow stuctures, resulting in moderate average total pressure and turbulence
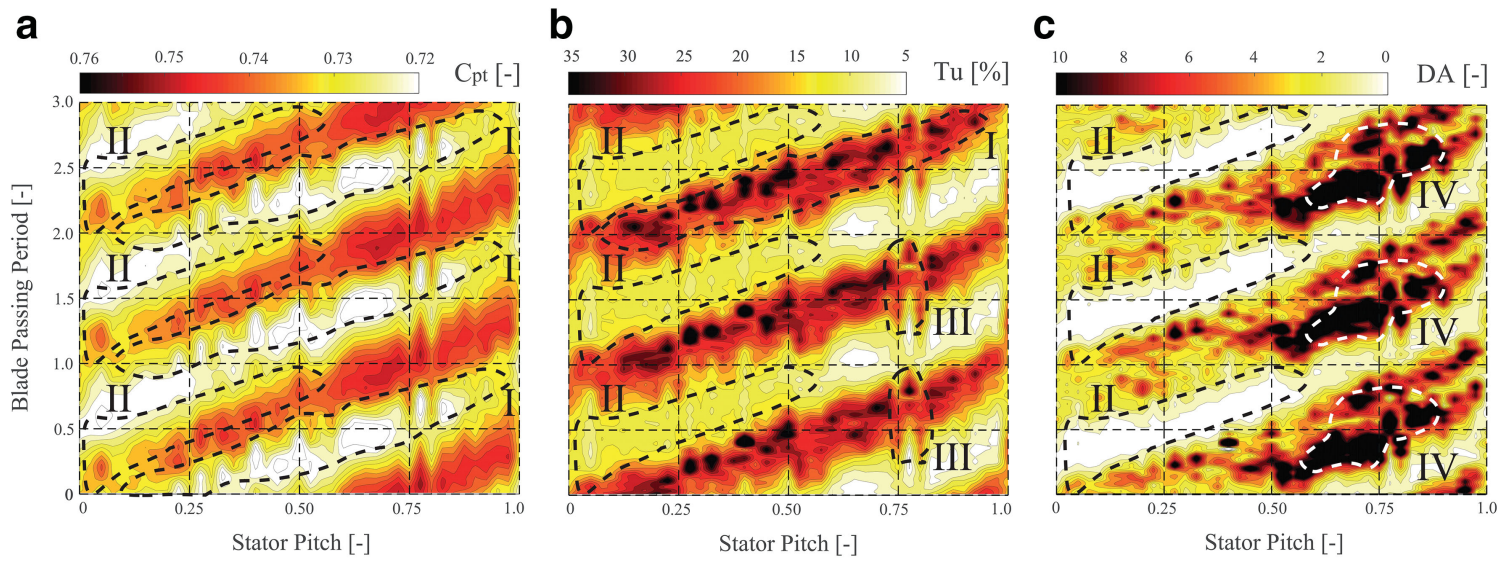

Figure 10. Time-space diagrams for total pressure coefficient (a), anisotropic turbulence (b) and Degree of Anisotropy DA (c) at $77 \%$ span. 
a

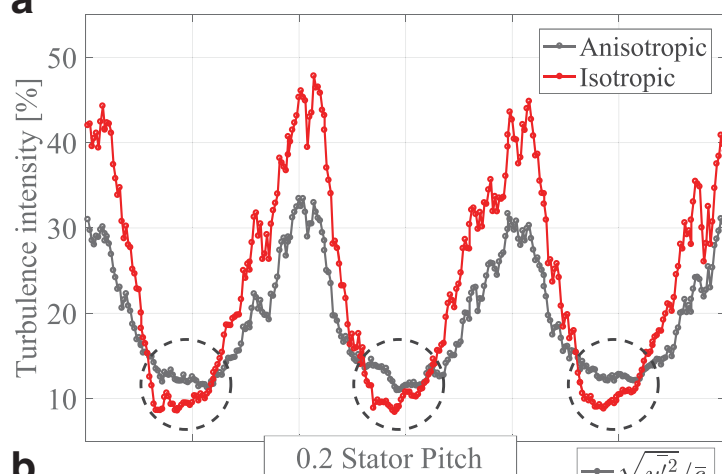

b

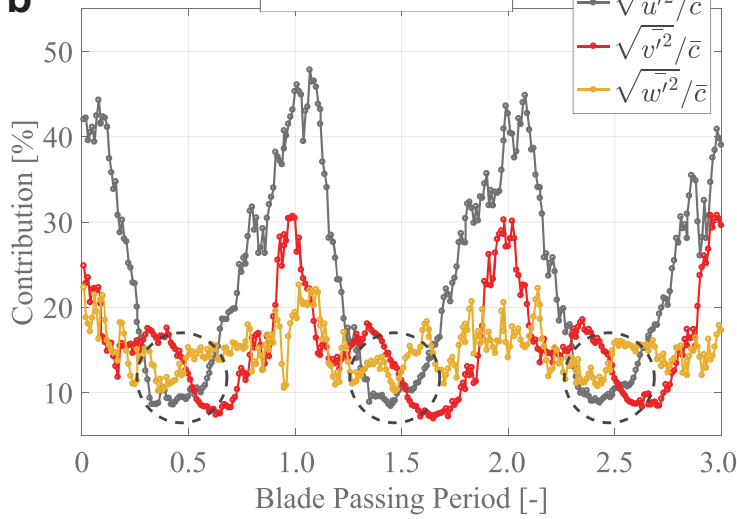

Figure 11. Turbulence intensity (a) and contribution of each velocity component to the overall turbulence level (b) at $77 \%$ span and 0.2 stator pitch. levels (Figure 8a, b zone D). In that zone, the cross-wise turbulence components tend to be nearly 4 times higher, compared to the streamwise direction.

In order to gain more insight into the contribution of each velocity components to the overall turbulence, the temporal variation of turbulence intensity (non- and isotropic) and the percentage contribution of each velocity components over three blade passing events are depicted in Figure 11a and Figure 11b, respectively, for a measurement point located at $77 \%$ span and 0.2 stator pitch. Figure $11 \mathrm{a}$ confirms that the assumption of isotropic turbulence does not hold in the context of rotating machinery flows.

The isotropic turbulence tends to overstimate the turbulence levels found in the secondary flow structures, whereas in regions weakly affected by secondary flow structures the opposite is true. This observation is in line with the work presented in (Porreca et al., 2007), even though their survey was conducted at the exit of a stator stage. As shown in Figure 11b, it can be observed that the contribution of both crosswise terms to the free-stream turbulence tends to be of the same order of magnitude, while across the tip passage vortex the meridional component contributes up to $10 \%$ more (in absolute values) compared to the vertical velocity component.

\section{Conclusions}

A novel 4-sensor fast response aerodynamic probe with a hemispherical tip diameter of $4 \mathrm{~mm}$ was successfully developed, calibrated and tested in a high-pressure axial turbine research facility. The probe demonstrated its reliability to perform accurate, time-averaged and -resolved measurements in the challenging environment of highly loaded, low aspect ratio turbine configuration. The main conclusions drawn from the presented work are summarized below:

- The newly developed FRAP-4S demonstrated similar behaviour with FRAP-2S, in terms of aerodynamic performance and dynamic response. Most importantly, the new probe enables a reduction of measuring time by a factor of 2.5 compared to the well-established FRAP-2S. This is directly translated to a reduction of operating time and thus cost, during development phase and testing of turbomachines.

- Comparative measurements with a miniature 5-hole probe of $0.9 \mathrm{~mm}$ in diameter were conducted at the exit of a rotor stage of an axial turbine. The deviation in measured yaw and $C_{p t}$ distribution between the FRAP-4S and the 5-hole probe remains on average within $8 \%$ and $0.5 \%$ over the entire span, respectively. Excellent agreement was found from 30 to $80 \%$ span and the largest deviations were observed close to the end-wall regions due to the four times larger tip diameter of the FRAP-4S resulting in larger blockage and spatial integration compared to the 5-hole probe. 
- The FRAP-4S enables the synchronous measurements of all stochastic velocity components as well as the complete Reynolds stress tensor. Using the probe, high levels of turbulence were measured across the entire plane at the rotor exit. Time-averaged turbulence intensity levels are of the order of $15 \%$, while in secondary flow regimes, as in the tip leakage, values as high as $25 \%$ were found. Moreover, the time-resolved turbulence results show a peak-to-peak variation of the turbulence intensity ranging from 5 to $35 \%$ over time.

- A high degree of anisotropy is also observed across the entire flow path, indicating the weakness of the isotropy assumption for the highly complex turbomachinery flow. At $77 \%$ span in relatively uniform flow regimes the stochastic fluctuations in the cross-wise direction dominate over the stream-wise components by a factor of 4, while the opposite stands for the tip passage vortex with turbulence levels up to ten times higher in the stream-wise direction.

\section{Nomenclature}

\section{Variables}

C Absolute velocity vector $[\mathrm{m} / \mathrm{s}]$

C Local free stream velocity $[\mathrm{m} / \mathrm{s}$ ]

$\mathrm{C}_{\mathrm{pt}}$ Normalized total pressure coefficient [-]

D Probe tip diameter $[\mathrm{mm}]$

$D A$ Degree of Anisotropy [-]

$f$ Frequency $[\mathrm{Hz}]$

$k \quad$ Reduced frequency [-]

$\mathrm{K}$ Aerodynamic coefficient [-]

M Mach number [-]

$\mathrm{p}$ Pressure $[\mathrm{Pa}]$

t Time [s]

Tu Turbulence intensity [\%]

$\mathrm{u}$ Stream-wise velocity component $[\mathrm{m} / \mathrm{s}$ ]

v Circumferential velocity component $[\mathrm{m} / \mathrm{s}]$

w Radial velocity component $[\mathrm{m} / \mathrm{s}]$

\section{Subscripts/superscripts}

' Stochastic (aperiodic) component

— Phase locked averaged

crit critical

iso isotropic

$s \quad$ static flow quantity

t stagnation flow quantity

\section{Greek}

$\gamma$ Pitch angle $\left[^{\circ}\right]$

$\varphi$ Yaw angle $\left[^{\circ}\right]$ 


\section{Abbreviations}

5HP Five-hole Pneumatic Probe

BPF Blade Passing Frequency

FRAP Fast Response Aerodynamic Probe

LEC Laboratory for Energy Conversion

PLA Phase Locked Averaged

V4S Virtual 4-Sensor

\section{Acknowledgements}

The authors would like to thank Dr Ilias Bosdas for his contribution at the beginning of this project. Moreover, the authors would like to acknowledge Dominic Hänni and Rainer Schädler for their active support during the measurement campaign in LISA facility. Finally, the authors would like to thank Mr Flori Alickaj for his support in the development of the new FRAP-4S measurement system.

\section{Funding sources}

None.

\section{Competing interests}

Alexandros C. Chasoglou declares that he has no conflict of interest. Michel Mansour declares that he has no conflict of interest. Anestis I. Kalfas declares that he has no conflict of interest. Reza S. Abhari declares that he has no conflict of interest.

\section{References}

Abhari R. S. (2006). Unsteady fluid dynamics of turbines: Perspective on possible directions to improve future engine designs. In: LaGraff J. E., Ashpis D. E., Martin L. G., Oldfield, and Gostelow J. P., editors. Proceedings of Minnowbrook V 2006 Workshop on Unsteady Flows in Turbomachinery, Volume NASA/CP 2006-214484, December 2006.

Ainsworth R. W., Miller R. J., Moss R. W., and Thorpe S. J. (2000). Unsteady pressure measurement. Measurement Science and Technology. 11 (7): 1055-1076. https://doi.org/10.1088/0957-0233/11/7/319.

Behr T., Kalfas A. I., and Abhari R. S. (2007). Unsteady flow physics and performance of a one-and-1/ 2-stage unshrouded high work turbine. Journal of Turbomachinery. 129 (2): 348-359. https://doi.org/10.1115/1.2447707.

Behr T., Kalfas A. I., and Abhari R. S. (2008). Desensitization of the flowfield from rotor tip-gap height by casing-air injection. Journal of Propulsion and Power. 24 (5): 1108-1116. https://doi.org/10.2514/1.34891.

Bosdas I., Mansour M., Kalfas A. I., and Abhari R. S. (2016). A fast response miniature probe for wet steam flow field measurements. Measurement Science and Technology. 27 (12): 1-17. https://doi.org/10.1088/0957-0233/27/12/125901.

Bosdas I., Mansour M., Kalfas A. I., and Abhari R. S. (2017). Experimental methods for performace and reliability of steam and gas turbines. Proceedings of the 1st Global Power and Propulsion Forum, Zurich, Switzerland, 16-18 January 2017. 1-11.

Brouckaert J. F. (2007). Fast response aerodynamic probes for measurements in turbomachines. Proceedings of the Institution of Mechanical Engineers, Part A: Journal of Power and Energy. 221 (6): 811-813. https://doi.org/10.1243/09576509jpe460.

Bubeck H. and Wachter J. (1987). Development and application of a high frequency wedge probe. ASME 1987 International Gas Turbine Conference and Exhibition, Anaheim, California, USA, 31 May-4 June 1987, International Gas Turbine Institute. 1-8. https://doi.org/10.1115/87-GT-216.

Camp T. R. and Shin H.-W. (1995). Turbulence intensity and length scale measurements in multistage compressors. Journal of Turbomachinery. 117 (1): 1-9. https://doi.org/10.1115/1.2835642.

Denton J. D. (1993). Loss mechanisms in turbomachines. Journal of Turbomachinery. 115 (4): 621. https://doi.org/ $10.1115 / 1.2929299$.

Gossweiler C. R. (1993). Sonden und Messsystem für Schnelle Aerodynamische Strömungsmessung mit Piezoresistiven Druckgebern. Dissertation Eidgenössische Technische Hochschule Zürich Nr. 10253, Zürich 1993. 
Heneka A. (1983). Entwicklung und Erprobung einer Keilsonde für instationäre dreidimensionale Strömungsmessungen in Turbomaschinen. PhD thesis, Universität Stuttgart.

Humm H. J., Gizzi W. P., and Gyarmathy G. (1994). Dynamic response of aerodynamic probes in fluctuating 3D flows. Proceedings of the 12th Symposium on Measuring Techniques for Transonic and Supersonic Flows in Cascades and Turbomachines, Academy of Sciences of the Czech Republic, Prague 1994. 1-15.

Humm H. J., Gossweiler C. R., and Gyarmathy G. (1995). On fast-response probes: Part 2-Aerodynamic probe design studies. Journal of Turbomachinery. 117 (4): 1-7. https://doi.org/10.1115/1.2836580.

Johansen E. S., Rediniotis O. K., and Jones G. (2001). The compressible calibration of miniature multi-hole probes. Journal of Fluids Engineering. 123 (1): 128. https://doi.org/10.1115/1.1334377.

Kerrebrock J. L., Epstein A. H., Haines D. M., and Thompkins W.T. (1974). The MIT Blowdown Compressor Facility. Journal of Engineering for Power. 96 (4): pp. 394-405. https://doi.org/10.1115/1.3445863.

Köppel P. D. (2000). Instationäre Strömung in Turbomaschinen: Analyse Zeitabhängiger Sondenmessungen. PhD thesis, ETH Zürich.

Kupferschmied P., Köppel P., Gizzi W., Roduner C., and Gyarmathy G. (2000b). Time-resolved flow measurements with fast-response aerodynamic probes in turbomachines. Measurement Science and Technology. 11 (7): 1036-1054. https://doi. org/10.1088/0957-0233/11/7/318.

Kupferschmied P., Köppel P., Gizzi W., Roduner C., and Gyarmathy G. (2000a). Time-resolved flow measurements with fast-response aerodynamic probes in turbomachines. Measurement Science and Technology. 11 (7): 1036-1054. https://doi. org/10.1088/0957-0233/11/7/318.

Lengani D., Paradiso B., and Marn A. (2012). A method for the determination of turbulence intensity by means of a fast response pressure probe and its application in a LP turbine. Journal of Thermal Science. 21 (1): 21-31. https://doi.org/ $10.1007 / s 11630-012-0515-8$.

Lenherr C., Kalfas A. I., and Abhari R. S. (2010). High temperature fast response aerodynamic probe. Journal of Engineering for Gas Turbines and Power. 133 (1): 11603. https://doi.org/10.1115/1.4001824.

Mansour M., Chokani N., Kalfas A. I., and Abhari R. S. (2008). Time-resolved entropy measurements using a fast response entropy probe. Measurement Science and Technology. 19 (11): 115401. https://doi.org/10.1088/0957-0233/19/11/115401.

Persico G., Gaetani P., and Paradiso B. (2008). Estimation of turbulence by single-sensor pressure probes. Proceedings of the XIX Biannual Symposium on Measuring Techniques in Turbomachinery, Rhode-St-Genèse, Belgium. 1-10.

Pfau A., Schlienger J., Kalfas A. I., and Abhari R. S. (2002). Virtual four sensor fast response aerodynamic probe (FRAP'). The 16th Symposium on Measuring Techniques in Transonic and Supersonic Flow in Cascades and Turbomachines. Cambridge, UK. 1-5.

Porreca L., Hollenstein M., Kalfas A. I., and Abhari R. S. (2007). Turbulence measurements and analysis in a multistage axial turbine. Journal of Propulsion and Power. 23 (1): 227-234. https://doi.org/10.2514/1.20022.

Regina K. (2015). High-Pressure Turbines With Novel Airfoils and End Walls Operating Under Engine Representative Aero-Thermodynamic Effects. PhD thesis, ETH Zürich.

Regina K., Basol A. M., Jenny P., Kalfas A. I., and Abhari R. S. (2013). Hot streak shaping and migration in an axial turbine. International Journal of Gas Turbine, Propulsion and Power Systems. 5 (1): 30-36.

Ruck G. (1988). Turbulence measurements with a high response pressure probe. Proceedings of 9th Symposium on Measuring techniques for transonic and supersonic flows in cascades and turbomachines, St. Catherine's College Oxford.

Ruck G. and Stetter H. (1990). Unsteady velocity and turbulence measurements with a fast response pressure probe. ASME 1990 International Gas Turbine and Aeroengine Congress and Exposition, Brussels, Belgium, 11-14 June 1990, International Gas Turbine Institute. 1-8. https://doi.org/10.1115/90-GT-232.

Schädler R., Kalfas A. I., Abhari R. S., Schmid G., auf dem Kampe T. and Prabhu S. B. (2017). Novel high-pressure turbine purge control features for increased stage efficiency. Journal of the Global Power and Propulsion Society. 1: p. 68MK5V. https://doi.org/10.22261/68MK5V.

Sieverding C. H., Arts T., Dénos R., and Brouckaert J.-F. (2000). Measurement techniques for unsteady flows in turbomachines. Experiments in Fluids. 28 (4): 285-321. https://doi.org/10.1007/s003480050390.

Treiber M., Kupferschmied P., and Gyarmathy G. (1998). Analysis of the error propagation arising from the measurements with a miniature pneumatic 5-hole probe. XIVth Symposium on Measuring Techniques for Transonic and Supersonic Flows in Cascade and Turbomachines.

Zimmermann T. W. and Wirsum M. (2016). Calibration and implementation of a trasient sub miniature 5-hole probe to determine complex flow structures in turbomachines. XXIII Biannual Symposium on Measuring Techniques in Turbomachinery Transonic and Supersonic Flow in Cascades and Turbomachines, Stuttgard. 1-10. 